\title{
Synergistic Antiproliferative Activity of the RAD51 Inhibitor IBR2 with Inhibitors of Receptor Tyrosine Kinases and Microtubule Protein $₫$
}

\author{
Peter J. Ferguson, Mark D. Vincent, and James Koropatnick \\ London Regional Cancer Program and Lawson Health Research Institute, London, Ontario, Canada (P.J.F., M.D.V., J.K.); and \\ Departments of Microbiology and Immunology (J.K.), Pathology (J.K.), Physiology and Pharmacology (J.K.), and Oncology (P.J.F., \\ M.D.V., J.K.), University of Western Ontario, London, Ontario, Canada
}

Received March 24, 2017; accepted October 20, 2017

\begin{abstract}
Although cancer cell genetic instability contributes to characteristics that mediate tumorigenicity, it also contributes to the tumorselective toxicity of some chemotherapy drugs. This synthetic lethality can be enhanced by inhibitors of DNA repair. To exploit this potential Achilles heel, we tested the ability of a RAD51 inhibitor to potentiate the cytotoxicity of chemotherapy drugs. 2-(Benzylsulfonyl)-1-(1H-indol-3-yl)-1,2-dihydroisoquinoline (IBR2) inhibits RAD51-mediated DNA double-strand break repair but also enhances cytotoxicity of the Bcr-Abl inhibitor imatinib. The potential for synergy between IBR2 and more drugs was examined in vitro across a spectrum of cancer cell lines from various tissues. Cells were exposed to IBR2 simultaneously with inhibitors of receptor tyrosine kinases, DNA-damaging agents, or microtubule disruptors. IBR2, at concentrations that inhibited proliferation
\end{abstract}

between $0 \%$ and $75 \%$, enhanced toxicity by up to $80 \%$ of imatinib and regorafenib (targets RAF and kit); epidermal growth factor receptor inhibitors erlotinib, gefitinib, afatinib, and osimertinib; and vincristine, an inhibitor of microtubule function. However, IBR2 antagonized the action of olaparib, cisplatin, melphalan, and irinotecan. A vincristine-resistant squamous cell line was not cross resistant to imatinib, but IBR2 and another RAD51 inhibitor (B02) enhanced imatinib toxicity in this cell line, its $\mathrm{HN}-5$ a parent, and the colon cancer line HT-29 by up to $60 \%$ and much better than verapamil, a P-glycoprotein inhibitor $(P<0.05)$. Given the disparate agents the functions of which are enhanced by IBR2, the mechanisms of enhancement may be multimodal. Whether RAD51 is common to these mechanisms remains to be elucidated, but it provides the potential for selectivity to tumor cells.

\section{Introduction}

The success of anticancer chemotherapy is often limited by the development of drug resistance by tumors, a phenomenon that evolves spontaneously due to the inherent genetic instability of cancer cells. This same feature renders cancer cells more sensitive than normal cells to many kinds of cytotoxic insult and contributes to the often limited selectivity of anticancer agents for tumor cells. However, some cancer cells have significant deficits in specific guardians of genomic maintenance, contributing to even greater sensitivity to agents that target particular enzymes or biochemical pathways. This specific hypersensitivity is referred to as synthetic lethality and has been exploited to treat tumors that develop

This work was supported by a grant from the Medical Oncology Research Fund, London Regional Cancer Program, London Health Sciences Centre, London, ON N6A 4L6, Canada.

Portions of this work were presented in abstract form: Ferguson PJ, Vincent MD, and Koropatnick J (2016) Synergistic anticancer activity of the targeted drugs imatinib, regorafenib, and gefitinib with the RAD51 inhibitor IBR2, in Proceedings of 28th EORTC-NCI-AACR Symposium on Molecular Targets and Cancer Therapeutics; 29 November-2 December, Munich, Germany.

https://doi.org10.1124/jpet.117.241661.

S This article has supplemental material available at jpet.aspetjournals.org. and/or progress as a result of specific lesions promoting genetic instability. For example, breast cancers that develop due to the absence of functional BRCA1 or BRCA2, important in homologous recombination-dependent DNA repair, are more sensitive to treatment with inhibitors of other DNA repair pathways, namely, inhibitors of poly(ADP-ribose) polymerase (PARP) (Bryant et al., 2005; Farmer et al., 2005).

Since tumors with inherent, pre-existing molecular alterations that provide synthetic lethal opportunity comprise only a small minority of cancer cases, induction of synthetic lethality in tumors without such pre-existing alterations would expand opportunities for chemotherapy success. Induced synthetic lethality would create selective sensitivity to specific drugs, allow use of lower doses, and spare toxicity to normal tissues. We have demonstrated induction of synthetic lethality by decreasing the levels of BRCA2 in cultured tumor cell lines, enhancing their sensitivity to inhibitors of PARP and other biochemical targets (Ferguson et al., 2013; Rytelewski et al., 2013, 2016). More recently, this form of induction includes downregulation of RAD51 to sensitize a variety of cancer cell lines to a plethora of anticancer drugs (Ferguson et al., 2016). RAD51 is an essential protein for homologous recombination-dependent DNA repair (Godin et al., 2016).

ABBREVIATIONS: 17-AAG, 17- $N$-allylamino-17-demethoxygeldanamycin; CML, chronic myeloid leukemia; EGFR, epidermal growth factor receptor; Hsp90, heat shock protein 90; IBR2, 2-(benzylsulfonyl)-1-(1H-indol-3-yl)-1,2-dihydroisoquinoline; PARP, poly(ADP-ribose) polymerase; Pgp, P-glycoprotein. 
Therefore, it was appropriate to determine the ability of a small molecule inhibitor of a DNA damage repair protein to enhance sensitivity to anticancer agents. Although there are currently no commercially available inhibitors of BRCA1 or 2, several inhibitors of RAD51 have been reported. 2-(Benzylsulfonyl)-1-(1H-indol-3-yl)-1,2-dihydroisoquinoline) (IBR2) was designed to specifically bind to and inhibit RAD51 (Lee et al., 2009). IBR2 downregulates the protein level and repair activity of RAD51, presumably inducing apoptosis through development of toxic double-strand DNA lesions leading to in vivo tumor growth inhibition (Zhu et al., 2013). IBR2 also enhanced sensitivity of K562 chronic myeloid leukemia (CML) cells to imatinib (Gleevec(C) (Zhu et al., 2013), an inhibitor of Bcr-Abl that also inhibits c-kit and platelet-derived growth factor receptor. The ability of IBR2 to enhance imatinib and multiple other anticancer agents was examined across a range of human tumor cell lines, since their dependence on various oncogenic drivers could lend evidence to the mechanism of the enhancement. IBR2 was tested in combination with regorafenib, which inhibits multiple molecules, including all of those inhibited by imatinib. Other drugs tested included inhibitors of epidermal growth factor receptor (EGFR) and microtubule function, and DNA-damaging agents. The findings indicate that IBR2 sensitizes cells to numerous agents. It is unclear whether RAD51 is directly involved in the enhancement of antiproliferative activity of these agents by IBR2. Some of the findings suggest that other targets for IBR2 may be involved.

\section{Materials and Methods}

Cells. The human head and neck squamous cell carcinoma cell line HN-5a was established at the University of Western Ontario from the gingival tumor of a patient not previously treated with chemotherapy or radiation (Lapointe et al., 1992). The multidrug-resistant P-glycoprotein (Pgp)-expressing cell line HN-5a/V15e was established as previously described (Ferguson et al., 2009).The small-cell lung cancer cell line H69 was provided by Dr. Susan Cole, Queen's University, Kingston, Canada (Slovak et al., 1993). All other cell lines were obtained from American Type Culture Collection (www.atcc.org). A clonal line of non-small-cell lung cancer cell line A549 (A549b) was propagated from a single cell.

Chemicals. IBR2 was obtained from Dr. Jiewen Zhu and Dr. WenHwa Lee, University of California-Irvine, Irvine, CA (Lee et al., 2009). Cisplatin was obtained from Mayne Pharma (Montreal, Canada). Olaparib, imatinib, osimertinib, and afatinib were purchased from Cedarlane Laboratories (Burlington, Canada). siRNAs targeting RAD51 mRNA (ON-TARGETplus) and BRCA2 mRNA (Rytelewski et al., 2013), and nontargeting sc-RNA No. 2 were obtained from Dharmacon/Thermo Fisher Scientific (Lafayette, CO). Anti-RAD51 siRNA 51-a targets mRNA sequence 5'-UAUCAUCGCCCAUGCAUCA-3' [coding region, bases 1169-1187 (NM_002875.4)]. All other compounds were obtained from Sigma-Aldrich (Oakville, Canada).

Other Materials. Fetal bovine serum and Lipofectamine 2000œ were purchased from Invitrogen/ThermoFisher (Unionville, Canada). Cell culture plastic-ware was obtained from Invitrogen/Fisher Scientific and VWR (Mississauga, Canada). Cell culture media and chemicals were obtained from commercial sources.

Proliferation Assays. Cells were maintained in minimum essential medium alpha plus $10 \%$ fetal bovine serum and penicillin $(50 \mathrm{U} / \mathrm{ml}) / \mathrm{streptomycin}(50 \mathrm{mg} / \mathrm{l})$ (growth medium). Cultures were incubated in a humidified atmosphere of $5 \% \mathrm{CO}_{2}$ at $37^{\circ} \mathrm{C}$. Rapidly proliferating cells were used to establish cultures of experimental cells, which were allowed to plate overnight in 96 -well plates or $25-\mathrm{cm}^{2}$ flasks prior to manipulation.
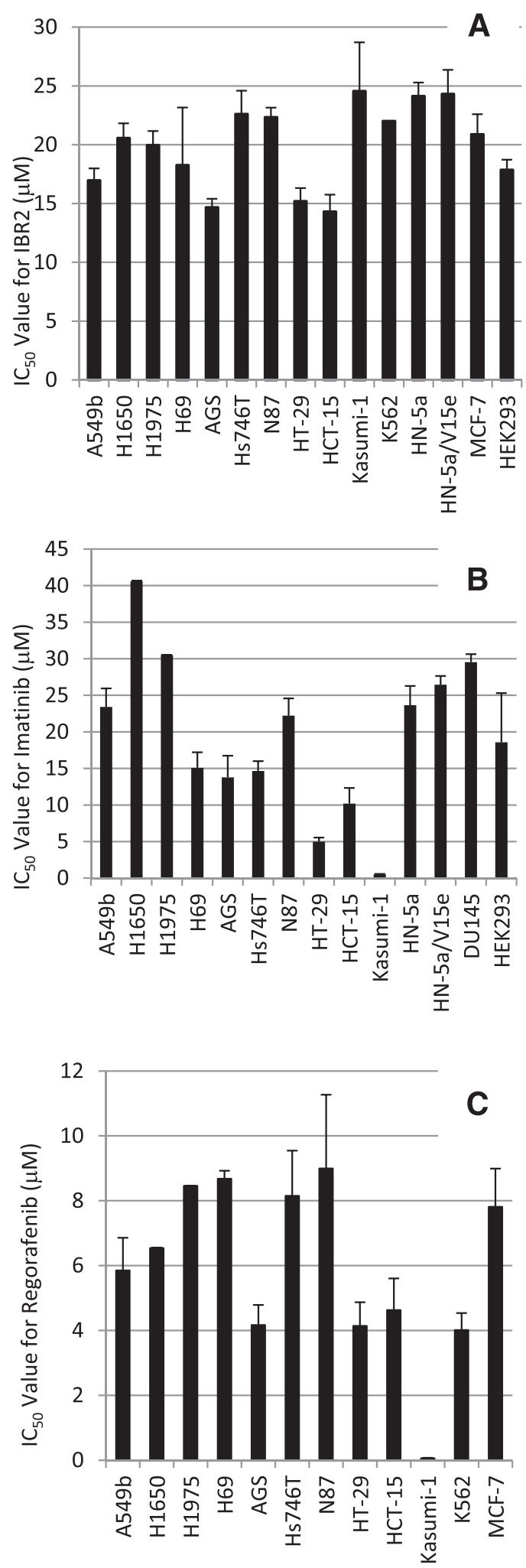

Fig. 1. The $\mathrm{IC}_{50}$ values for IBR2, imatinib, and regorafenib tested against a range of cell lines in vitro. Cells were grown in 96-well plates and exposed to a range of concentrations of drug. Relative cell density was determined by a viability assay (alamarBlue or neutral red). The $\mathrm{IC}_{50}$ value was interpolated from plotted data. Bars represent the mean values of between 3 and 29 determinations, except $n=1$ for K562, (A); H1650 and H1975 (B and C); error bars, S.D. 
Exposure to Chemotherapy Drug Plus IBR2, Verapamil, or B02. Cells were grown in 96 -well plates and treated with simultaneous exposure to the drugs at the concentrations indicated. The relative cell density was determined after 4 days by viability staining (alamarBlue $\odot$ or neutral red). The relative density of cells treated with both agents was normalized to the density observed for the respectively treated second agent control (IBR2, verapamil, or B02). The $\mathrm{IC}_{50}$ value was interpolated from plotted data. The change in the $\mathrm{IC}_{50}$ value of a chemotherapy drug caused by the presence of the second agent was determined as a percentage of the $\mathrm{IC}_{50}$ value of the first drug alone (Supplemental Fig. 1). Enhancement of cytotoxicity is indicated by a decrease in the $\mathrm{IC}_{50}$ value. This methodology, previously reported (Ferguson et al., 2009; Blake et al., 2017), provides a quantitative, concentration-dependent representation of the magnitude of the drug interaction.

Antisense Treatment. Cells were grown in $25-\mathrm{cm}^{2}$ flasks and allowed to plate overnight. siRNA was introduced into cells with the use of Lipofectamine 2000 , mixed at a ratio of $1.25 \mu \mathrm{g} / \mathrm{ml}$ per $10 \mathrm{nM}$ siRNA in serum-free medium. The mixture was prepared at $5 \times$ the final concentration to which cells were exposed, such that $0.5 \mathrm{ml}$ was added to $2 \mathrm{ml}$ of medium in which cells were plated. After incubating at room temperature for 20 minutes, according to manufacturer's instructions, the nucleic acids were added to the cell medium, and incubated $\left(37^{\circ} \mathrm{C}\right)$ on the cells for 4 hours, after which a second volume of medium was added. Cells were then incubated for 20 hours. Following this incubation, the siRNA-containing medium was replaced with drug-free medium. If the cells were to be exposed to a drug, for the purposes of determining whether inhibitory activity was enhanced by the antisense pretreatment, the drug was added at this time. At this point, replicate flasks from the antisense treatment were used to enumerate cell content, since this varied among treatments over the initial 24-hour exposure. This was done such that the effect of the second treatment could be ascertained based on the cell population that was present at the time of initiation of exposure. Exposure to the second drug was initiated by addition of 0.2 -volume of a drug preparation, at a $6 \times$ final concentration in growth medium, to the fresh, drug-free medium on the cells ( $1 \mathrm{ml}$ of drug into $5 \mathrm{ml}$ of medium). Following further incubation of 4 days, the proliferation of the treated cells (fold increase in cell number) was calculated as a percentage of that of control cells. Cell numbers were enumerated on an electronic particle counter (Coulter Z1; Beckman Coulter, Mississauga, Canada).

Flow Cytometry. A549b cells were incubated in IBR2 for 24 hours, followed by harvesting with trypsin, washing with phosphate-buffered saline, and fixing in 70\% ethanol. Fixed cells were stained with propidium iodide and analyzed with a flow cytometer (10,000 events) (BD LSR II; BD Biosciences, Mississauga, Canada) in the London Regional Flow Cytometry Facility, Robarts Research Institute, Western University, London, Canada. The percentage of cells in each of G1, S, and G2/M phases was determined using the curve-fitting program FlowJo (FlowJo LLC, Ashland, OR).

Statistical Evaluation. Differences between data sets were compared using a two-tailed Student's $t$ test. Significance levels were set a priori at $P<0.05$.

\section{Results}

To determine if IBR2 could have a broader usage to enhance the antitumor effect of chemotherapy drugs, the combination of IBR2 plus a range of drugs was tested against a panel of
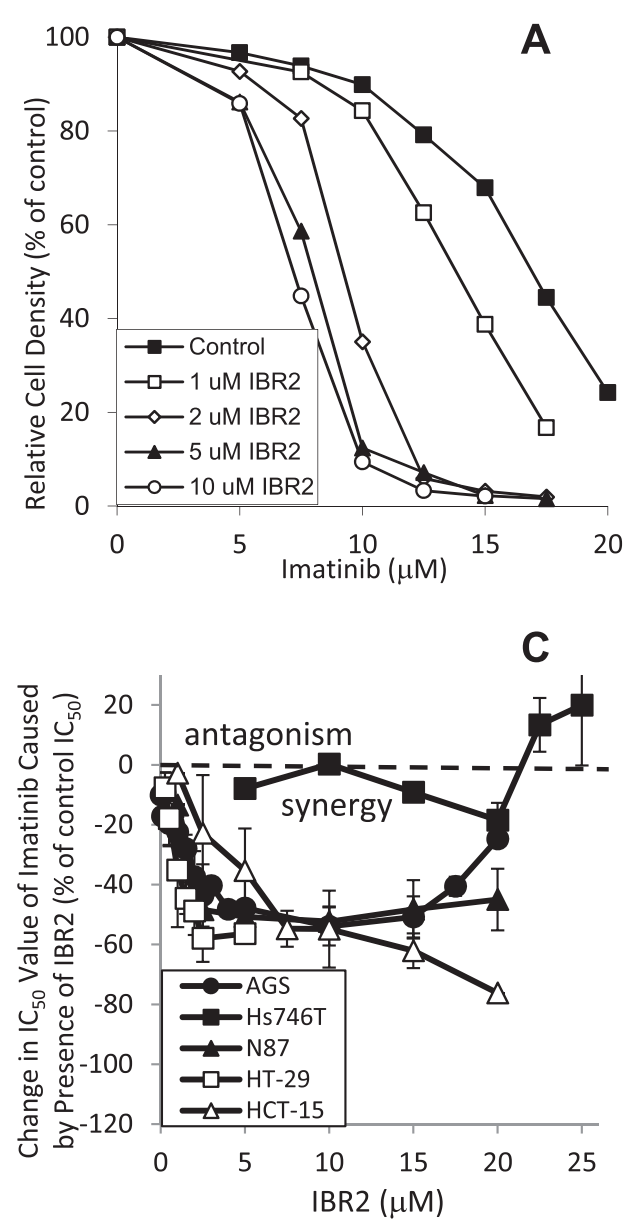

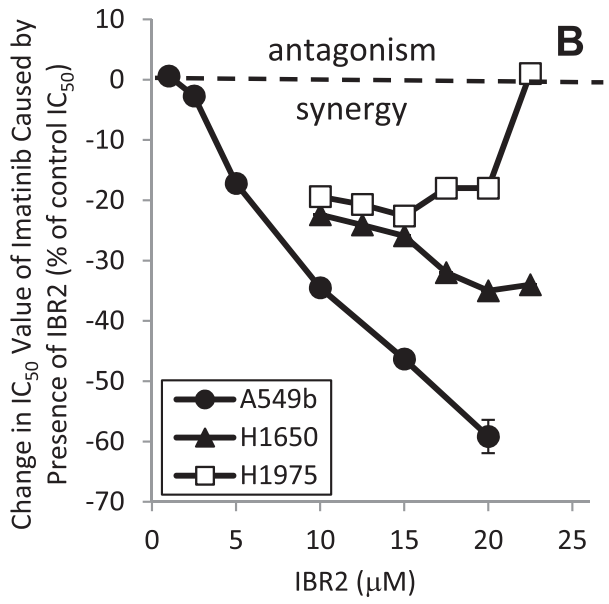

D

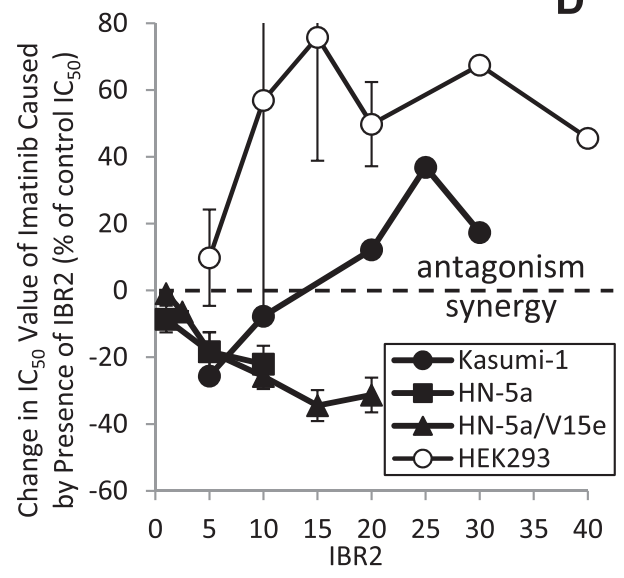

Fig. 2. Quantitation of enhancement of cytotoxicity of imatinib by IBR2. (A) Representative experiment demonstrating the ability of IBR2 to enhance the antiproliferative activity of imatinib against the AGS gastric carcinoma cell line. Cells were grown in 96-well plates, and treated with simultaneous exposure to IBR2 and the drugs at the concentrations indicated. The relative cell density was determined after 4 days by viability staining. The relative density of cells treated with both agents was normalized to the density observed for the respectively treated IBR2 control. The $\mathrm{IC}_{50}$ value was interpolated from plotted data. The change in the $\mathrm{IC}_{50}$ value caused by the presence of IBR2 was determined as a percentage of the $\mathrm{IC}_{50}$ value of imatinib in the absence of IBR2; therefore, enhancement of cytotoxicity (synergistic drug interaction), exemplified by a decrease in the $\mathrm{IC}_{50}$ value, manifests as a negative value. These values were determined over a range of concentrations of IBR2 in a variety of cell lines, and compiled to create (B-D) enhancement of imatinib cytotoxicity by IBR2 in non-small-cell lung cancer cell lines (B), gastric and colon carcinoma cell lines (C), and CML and head and neck squamous cell carcinoma and kidney cell lines (D). 
cancer cell lines derived from tissues that included lung, head and neck, gastric (stomach), breast, colon, prostate, and hematopoietic cells (acute myeloid leukemia and CML). The cell lines expressed a range of different oncogenic drivers, including chimeric Bcr-Abl (K562), mutations of k-RAS (A549b, AGS, and HCT-15) or b-Raf (HT-29), and mutant (Kasumi-1) or overexpressed c-Kit (H69), although some cell lines were of less descript etiology (Hs746T, N87, MCF-7, and DU145) (information from American Type Culture Collection, www.atcc.org). A virally transformed, human embryonic kidney cell line (HEK293) was also included in several of the analyses. Sensitivity to IBR2 was very similar across all of the cell lines studied, in which the $\mathrm{IC}_{50}$ values ranged between 15 and $25 \mu \mathrm{M}$ (Fig. 1; Supplemental Fig. 1). Sensitivity to imatinib and regorafenib ranged more broadly, and Kasumi-1, which expresses mutant c-Kit, was highly sensitive to both of these. In A549b cells, IBR2, at concentrations that inhibited proliferation by greater than $50 \%$, induced a significant accumulation of cells in G1, at the expense of a decrease of population in S and G2/M (Supplemental Fig. 2). For the drug-combination studies described subsequently, IBR2 was used at concentrations that inhibited proliferation by up to $75 \%$.

When IBR2 was combined with imatinib, as previously demonstrated in K562 cells (Zhu et al., 2013), the antiproliferative activity of imatinib was enhanced by $40 \%-60 \%$ in many of the cell lines (Fig. 2). Figure 2A is an example of the shift of the dose-response curves to lower imatinib concentrations in the presence of IBR2, in a concentration-dependent manner (AGS stomach cancer cell line in this example). The amount by which the curves were shifted was quantified for each curve in individual experiments as a function of the change in $\mathrm{IC}_{50}$ value (demonstrated in Supplemental Fig. 3). Other representative experiments, demonstrating the leftward shift in the dose-response curve, are presented in Supplemental Figs. 4-6. It is noteworthy that not only were the curves shifted to the left, but in most instances there were concentrations of IBR2 and drug that completely inhibited proliferation; as single agents, these concentrations inhibited proliferation by only $20 \%-60 \%$, and often by only $20 \%-40 \%$ (Supplemental Figs. 1 and 4-6). Although such extensive inhibition was observed in each experiment, it is cumbersome to report for the large number of combinations of drug concentrations. Therefore, the shift in $\mathrm{IC}_{50}$ value was chosen for analysis of the effects demonstrated herein because it is readily quantifiable and is reproducible between experiments. The amount of change in the $\mathrm{IC}_{50}$ value, calculated as a percentage of that $\mathrm{IC}_{50}$ value, was determined for a variety of concentrations of IBR2 against 12 of the cell lines (Fig. 2, B-D). IBR2 was not as effective at enhancing imatinib cytotoxicity in some cell lines as it was in others, indicating that there was some cell specificity to the degree of enhancement.

To determine whether IBR2 could also enhance cytotoxicity of other chemotherapy drugs, it was tested in combination with agents that have a variety of biochemical targets. Regorafenib targets RAF, kit, and a variety of growth factor and angiogenic receptor kinases. IBR2 enhanced the antiproliferative activity of regorafenib by up to $60 \%-80 \%$ in all of the cell lines tested except for Kasumi-1 (Fig. 3; Supplemental Fig. 5), which was already very sensitive to regorafenib (and reflective of a similar situation with response to imatinib in this cell line).
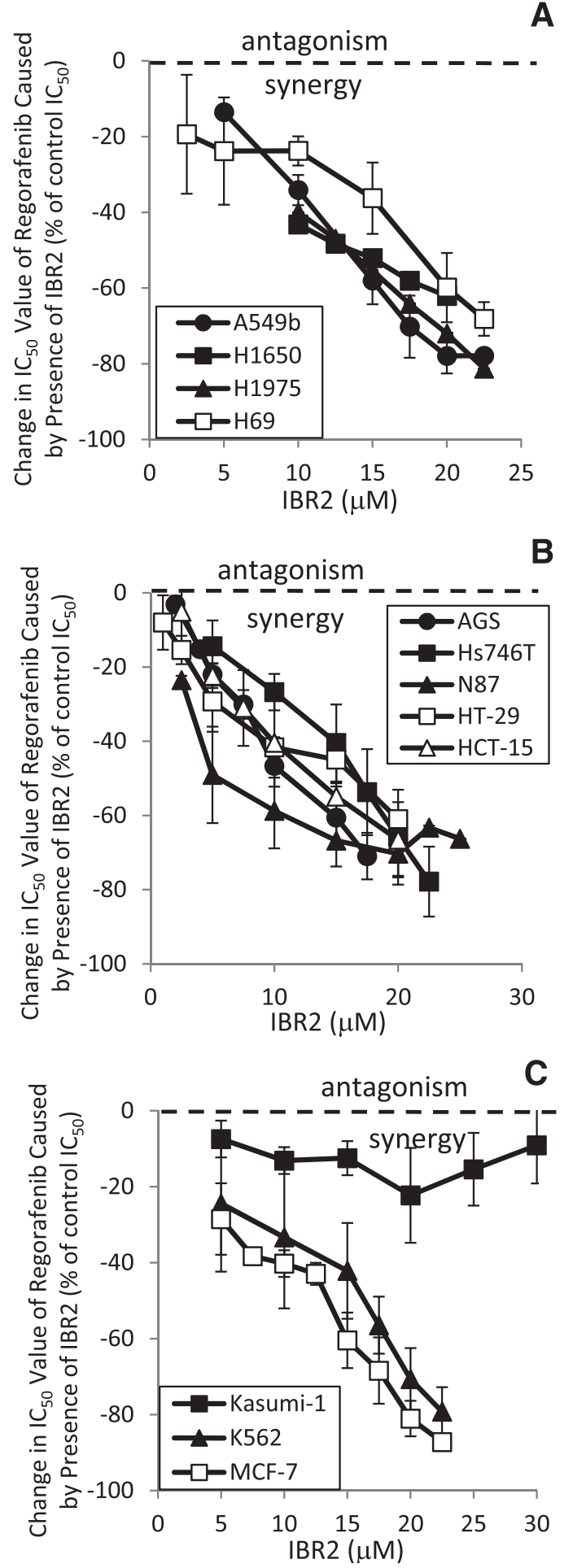

Fig. 3. Enhancement of cytotoxicity of regorafenib by IBR2. Cells were grown in 96-well plates, and treated with simultaneous exposure to the drugs at the concentrations indicated. The relative cell density was determined after 4 days by viability staining. The relative density of cells treated with both agents was normalized to the density observed for the respectively treated $\mathrm{IBR} 2$ control. The $\mathrm{IC}_{50}$ value was interpolated from plotted data. The change in the $\mathrm{IC}_{50}$ value caused by the presence of IBR2 was determined as a percentage of the $\mathrm{IC}_{50}$ value of regorafenib in the absence of IBR2. Negative values indicate synergy. These values were determined over a range of concentrations of IBR2 in a variety of cell lines, and compiled to create (A-C) enhancement of regorafenib cytotoxicity by IBR2 in non-small-cell and small-cell lung cancer cell lines (A), gastric and colon carcinoma cell lines (B), and leukemic and breast cancer cell lines (C). 
EGFR was chosen as a different biochemical target against which to determine whether its inhibitors could be enhanced by IBR2. For this assay, three non-small-cell lung cancer cell lines were used since this tumor type is commonly treated with EGFR inhibitors clinically but usually relapses with resistance to the drug. A variety of responses was observed when IBR2 was combined with different EGFR inhibitors (Fig. 4; Supplemental Fig. 6). Although IBR2 enhanced gefitinib toxicity against all three cell lines, there was no interaction with afatinib in A549b cells or erlotinib in H1975 cells, and there was even antagonism between IBR2 and erlotinib against A549b cells. Erlotinib tends to cause a plateau in proliferation at approximately $50 \%$ at concentrations of $10 \mu \mathrm{M}$ and above (tested to $50 \mu \mathrm{M}$ ), which is indicative of a cytostatic effect. This may account for the apparent antagonism when combined with IBR2. Against HEK293, although there was a small amount of enhancement of gefitinib and osimertinib by lower concentrations of IBR2, there was strong antagonism at higher concentrations that were synergistic against the cancer cell lines.

Based on limited information that imatinib may be a substrate for a multidrug transport protein (Radujkovic et al., 2005), there existed the possibility that IBR2 was acting to enhance drug activity by inhibiting a drug exporter, such as Pgp, thus increasing the intracellular drug accumulation. Therefore, the effect of combining IBR2 with imatinib was compared with the Pgp inhibitor verapamil against four different cell lines, including one cell line that overexpresses Pgp and is 3-fold resistant to vincristine (HN-5a/V15e) (Fig. 5) (Ferguson et al., 2009). As another comparison with these combinations, a second RAD51 inhibitor, B02 (Huang et al., 2012), was tested. Against all four cell lines tested, including HN-5a/V15e, IBR2 and B02 were more effective than verapamil at enhancing the antiproliferative activity of imatinib. It is also noteworthy that $\mathrm{HN}-5 \mathrm{a} / \mathrm{V} 15 \mathrm{e}$ is not cross resistant to imatinib (Fig. 1).

In combination with vincristine against these same four cell lines (Fig. 6), IBR2, B02, and verapamil exhibited similar enhancement at most concentrations with the following exceptions: in HT-29 cells, IBR2 was not as effective as B02 and verapamil at concentrations of $5 \mu \mathrm{M}$ and higher, but IBR2 and B02 were more effective than verapamil at $2 \mu \mathrm{M}$ and lower; as expected in vincristine-resistant $\mathrm{HN}-5 \mathrm{a} / \mathrm{V} 15 \mathrm{e}$ (since

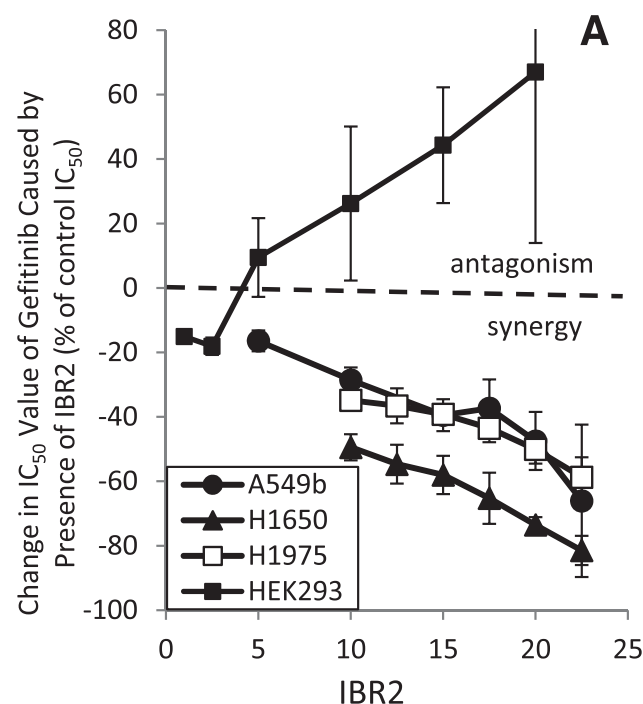

C

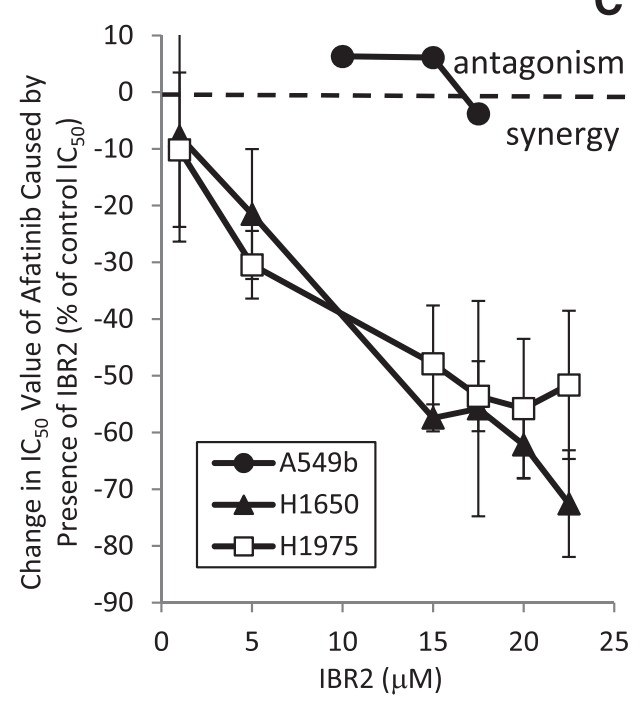

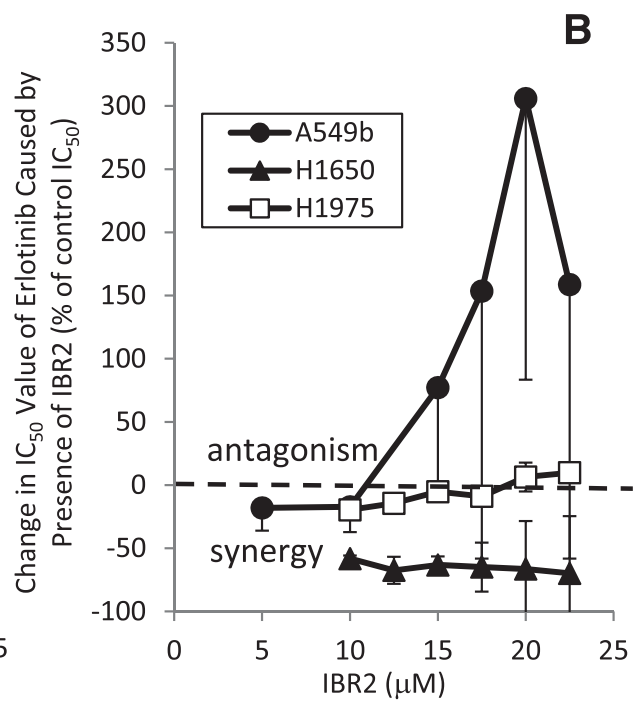

D

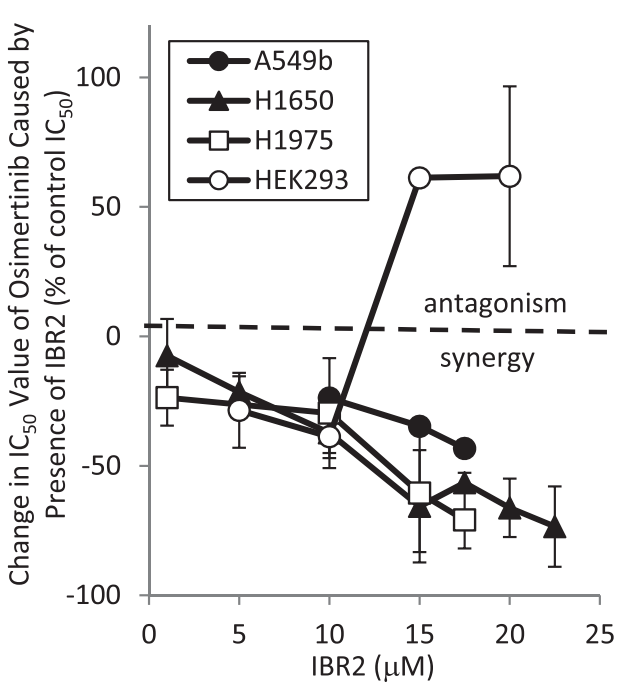

Fig. 4. Enhancement of inhibitors of EGFR in non-small-cell lung cancer cell lines by IBR2. Cells were grown in 96 -well plates, and treated with simultaneous exposure to IBR2 and the drugs at the concentrations indicated. The relative cell density was determined after 4 days by viability staining. The relative density of cells treated with both agents was normalized to the density observed for the respectively treated IBR2 control. The $\mathrm{IC}_{50}$ value was interpolated from plotted data. The change in the $\mathrm{IC}_{50}$ value caused by the presence of IBR2 was determined as a percentage of the $\mathrm{IC}_{50}$ of EGFR inhibitor in the absence of IBR2. Negative values indicate synergy. These values were determined over a range of concentrations of IBR2 in combination with gefitinib (A), erlotinib (B), afatanib (C), and osimertinib (D). The $\mathrm{IC}_{50}$ values $(\mu \mathrm{M})$ for gefitinib, erlotinib, afatanib, and osimertinib for A549b were $14.3 \pm 2.9(6), 7.2 \pm 1.2(6)$, 4.15 (1), and 6.16 (1), respectively; for H1650 were $11.2 \pm 1.4(3), 18.7 \pm 3.7$ (3), $1.83 \pm 0.22$ (3), and $3.53 \pm 0.11$ (3), respectively; and for H1975 were $13.8 \pm$ $1.0(3), 9.7 \pm 0.3(3), 0.38 \pm 0.08(3)$, and $0.034 \pm 0.013$ (3), respectively. For HEK293, the $\mathrm{IC}_{50}$ values $(\mu \mathrm{M})$ were $8.88 \pm 0.45$ (3) for gefitinib and $2.33 \pm 1.14$ (3) for osimertinib. 

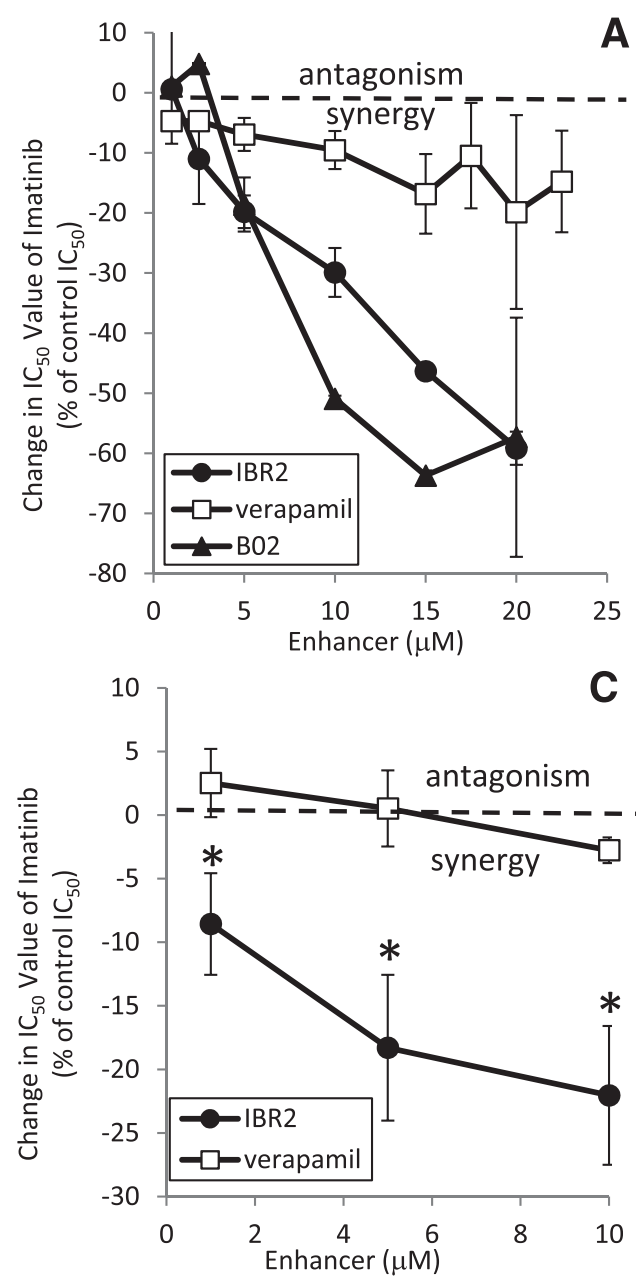

A
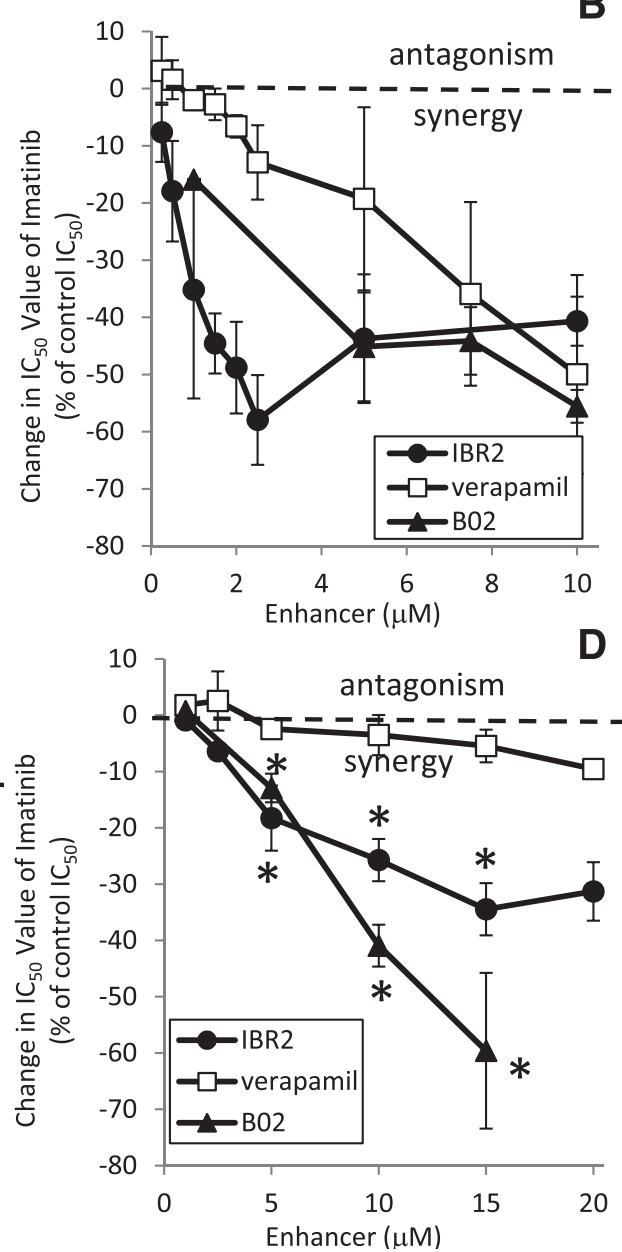

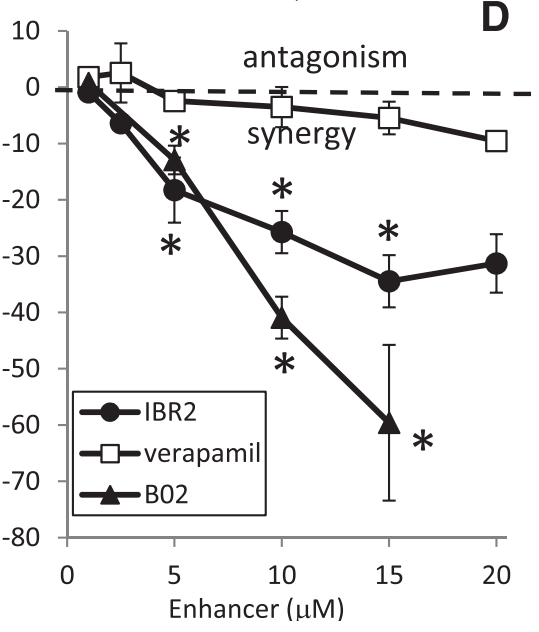

Fig. 5. Comparison of enhancement of imatinib cytotoxicity caused by IBR2, verapamil, or B02 (enhancers). Cells were grown in 96-well plates, and treated with simultaneous exposure to the drugs at the concentrations indicated. The relative cell density was determined after 4 days by viability staining. The relative density of cells treated with both agents was normalized to the density observed for the respectively treated enhancer control. The $\mathrm{IC}_{50}$ value of imatinib was interpolated from plotted data, as indicated in Fig. 1. The change in the $I_{50}$ value caused by the presence of enhancer was determined as a percentage of the $\mathrm{IC}_{50}$ value of imatinib in the absence of enhancer. Negative values indicate synergy. These values were determined over a range of concentrations of enhancer plus imatinib against carcinoma cell lines A549b (A), HT-29 (B), HN-5a (C), and HN-5a/V15e (D). Points are the mean values of 3-6 determinations [with error bars (S.D.)] or are from single experiments (no error bars); ${ }^{*} P<0.05$. The $\mathrm{IC}_{50}$ values for IBR2 and imatinib for these cell lines are indicated in Fig. 1. The $\mathrm{IC}_{50}$ values $(\mu M)$ for verapamil for the respective cell lines (above) are $88.7 \pm$ 0.9 (3), $17.6 \pm 0.7$ (5), $89.4 \pm 7.7$ (3), and $99.2 \pm 1.2(2)$. The $\mathrm{IC}_{50}$ values $(\mu \mathrm{M})$ for B02 for the respective cell lines are $11.8 \pm$ $2.1(6), 10.4 \pm 2.1(8), 12.2 \pm 0.9(4)$, and $15.0 \pm 5.4(3)$. verapamil is a classic inhibitor of Pgp), verapamil enhanced vincristine cytotoxicity better than IBR2 and B02, especially at lower concentrations.

Antisense knockdown of BRCA2 enhanced the antiproliferative activity of the PARP inhibitor olaparib by $50 \%$ (Rytelewski et al., 2016; Supplemental Fig. 7A), and similarly antisense siRNA against RAD51 also sensitized cell lines to inhibition by olaparib (Fig. 7, A and C). In contrast: 1) knockdown of BRCA2 had no effect on cytotoxicity of IBR2 (Supplemental Fig. 7B); 2) the combination of IBR2 and olaparib was antagonistic (Fig. 7B) or additive only (Supplemental Fig. 8), indicative of a possible interaction that is required between PARP and RAD51 in order for olaparib to incur DNA damage; and 3) anti-RAD51 siRNA, at concentrations that greatly enhanced olaparib toxicity, only slightly enhanced toxicity of drugs with which IBR2 was synergistic, and in some combinations was antagonistic (Fig. 7D). The enhancement of drug toxicity by IBR2 demonstrated specificity to certain drug classes, and did not include those that directly damage DNA. IBR2 was not able to enhance cytotoxicity of paclitaxel, irinotecan, melphalan, or cisplatin (Supplemental Fig. 9).

\section{Discussion}

IBR2 is among several recently developed RAD51 inhibitors (Huang et al., 2012; Zhu et al., 2013; Balbous et al., 2016; Lv et al., 2016), one of which enhances cell sensitivity to
X-irradiation (Lv et al., 2016). Another, B02, when combined with a PARP inhibitor, synergistically enhances the DNAdamaging agent methylmethane sulfonate (Huang et al., 2012). B02 was chosen for comparison purposes in the current study to help demonstrate whether the synergistic interactions between IBR2 and chemotherapy drugs could be attributable to a direct effect on RAD51.

IBR2 enhanced cytotoxicity of anticancer agents having a variety of biochemical targets, including imatinib (targets BcrAbl, c-kit, and platelet-derived growth factor receptor), regorafenib (RAF, kit, and various growth factor and angiogenic receptor kinases), inhibitors of EGFR (erlotinib, gefitinib, afatinib, and osimertinib), and vincristine (microtubules). Although $\mathrm{IC}_{50}$ values were decreased by up to $80 \%$, inhibition of proliferation was $95 \%-100 \%$ at many drug concentrations that alone were not substantially inhibitory (e.g., see Supplemental Figs. 4-6). Therefore, IBR2-drug combinations have the potential to fully inhibit tumor growth in vivo at doses that are minimally inhibitory on their own. For purposes of data interpretation, the choices of drug and enhancing agent (or second agent) are arbitrary, and the numbers could be used to indicate that imatinib, for example, enhanced IBR2 cytotoxicity. Because the concentrations of IBR2 used in these combinations inhibited proliferation between $0 \%$ and $75 \%$ as a single agent, there were instances in which the synergy between the two agents could be a result of enhancement of IBR2 toxicity (discussed subsequently). However, in most 
A

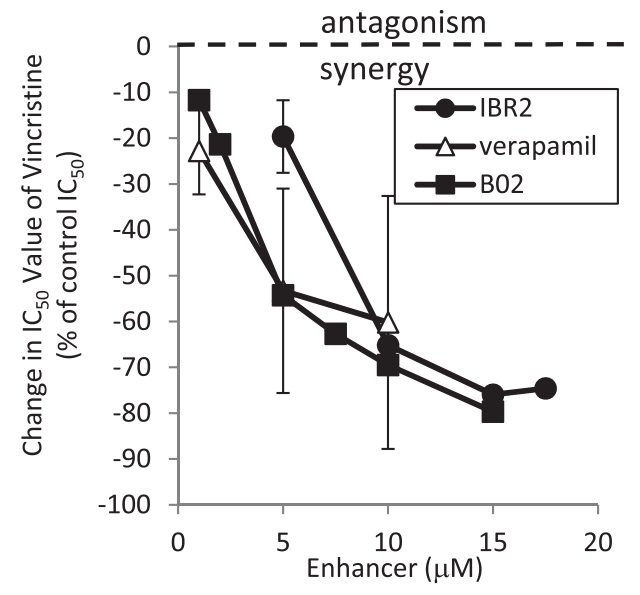

C

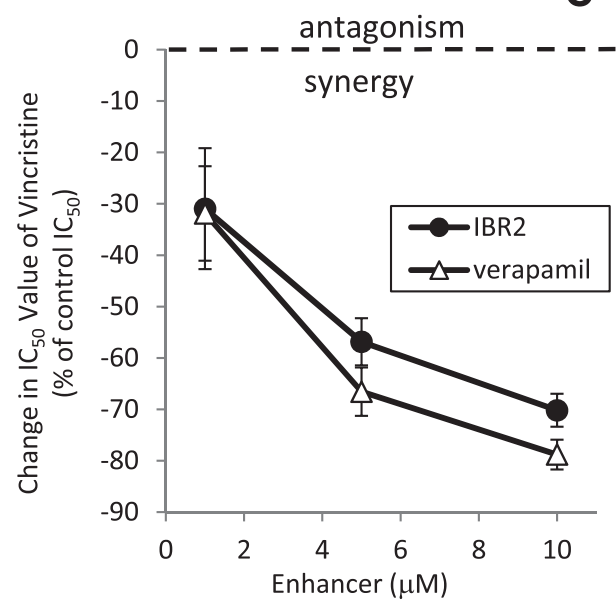

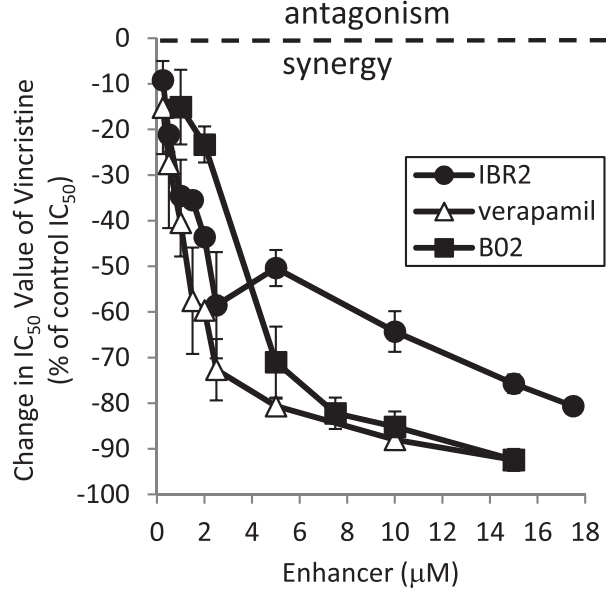

D

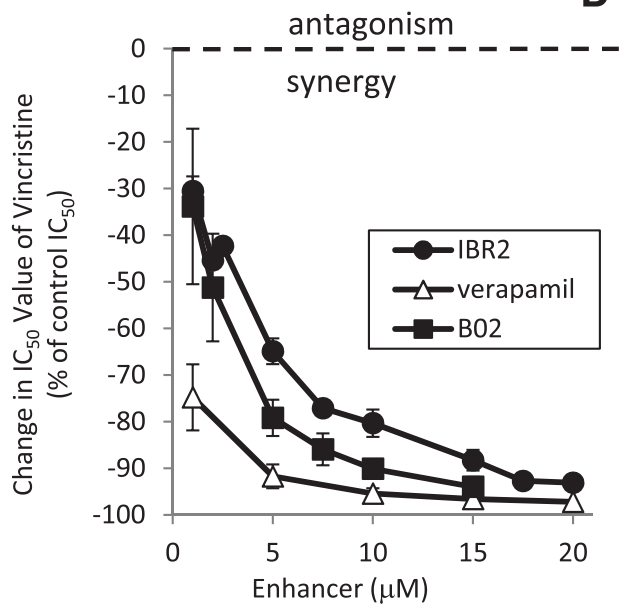

Fig. 6. Comparison of enhancement of vincristine cytotoxicity caused by IBR2, verapamil, or B02 (enhancers). Cells were grown in 96-well plates, and treated with simultaneous exposure to the drugs at the concentrations indicated. The relative cell density was determined after 4 days by viability staining. The relative density of cells treated with both agents was normalized to the density observed for the respectively treated enhancer control. The $\mathrm{IC}_{50}$ value of vincristine was interpolated from plotted data. The change in the $\mathrm{IC}_{50}$ value caused by the presence of enhancer was determined as a percentage of the $\mathrm{IC}_{50}$ value of vincristine in the absence of enhancer. Negative values indicate synergy. These values were determined over a range of concentrations of enhancer plus vincristine against carcinoma cell lines A549b (A), HT-29 (B), HN-5a (C), and HN-5a/V15e (D). Points are the mean values of 2-6 determinations [with error bars (S.D.)] or are from single experiments (no error bars). The $\mathrm{IC}_{50}$ values $(\mathrm{nM})$ for vincristine for each of the cell lines are $4.34 \pm 0.45(n=9), 3.61 \pm$ $0.56(15), 4.36 \pm 0.92(6)$, and $11.7 \pm 3.3$ (12), respectively. The $\mathrm{IC}_{50}$ values for IBR2 for these cell lines are indicated in Fig. 1. The $\mathrm{IC}_{50}$ values for verapamil and B02 are listed in the legend for Fig. 5. combinations, IBR2, verapamil, and B02 were considered as the enhancing agent for two reasons: 1) they were tested in combination with many different chemotherapy drugs, and were designated as the second agent for purposes of clarity and to facilitate comparisons between experiments; and 2) synergy was observed in many instances when the second agent was used at nontoxic concentrations.

The enhancement of cytotoxicity by IBR2 was not a broadspectrum, nonspecific effect. IBR2 sometimes had no effect on the drug, in which case the change in $\mathrm{IC}_{50}$ value induced by IBR2 was zero. In some instances IBR2 interfered with or antagonized the toxicity of the drug, in which case the change in $\mathrm{IC}_{50}$ value was positive (for examples, see Supplemental Fig. 9). Also, IBR2 did not enhance cytotoxicity of imatinib or regorafenib in Kasumi-1 cells, which are inherently sensitive to these agents.

The differences in the IBR2/drug interactions between the different drugs used indicate mechanistic specificity of the interaction, although at this point it is not possible to distinguish the basis of the selectivity. The aforementioned results and other evidence suggest that IBR2 may have several modes of action that confer its ability to enhance cytotoxicity of a variety of anticancer agents:

1. IBR2 enhanced cytotoxicity of drugs with disparate biochemical targets, including signal transduction proteins belonging to different pathways, and the microtubule disruptor vincristine (but not paclitaxel, except in the multidrug-resistant cell line). RAD51 is not linked directly with any of these functions.

2. In K562 cells (mutant c-Kit and Bcr-Abl expression), IBR2 enhanced regorafenib, which targets c-Kit. c-Kit and RAD51 are both client proteins of heat shock protein 90 (Hsp90), as are Bcr-Abl, Akt, and BRCA1 (Noguchi et al., 2006; Smyth et al., 2012; Suhane et al., 2015; Jiang et al., 2017). In Kasumi-1 cells (normal Abl and mutant c-Kit), which are hypersensitive to imatinib and regorafenib (Fig. 1; Beghini et al., 2005), IBR2 conferred minimal enhancement.

3. Given that IBR2 inhibits the protein expression and activity of an essential DNA repair protein, it was expected that IBR2 would enhance cytotoxicity of drugs that directly damage DNA (melphalan, cisplatin, and irinotecan). The fact that this was not the case indicates that a secondary mechanism of repair may compensate for inhibition of RAD51 (Wiegmans et al., 2016), but this mechanism does not compensate for the effects that enhance toxicity of non-DNAdamaging agents.

4. Since IBR2 enhanced vincristine cytotoxicity, a possible interaction with Pgp was investigated. The Pgp-overexpressing, multidrug-resistant cell line 


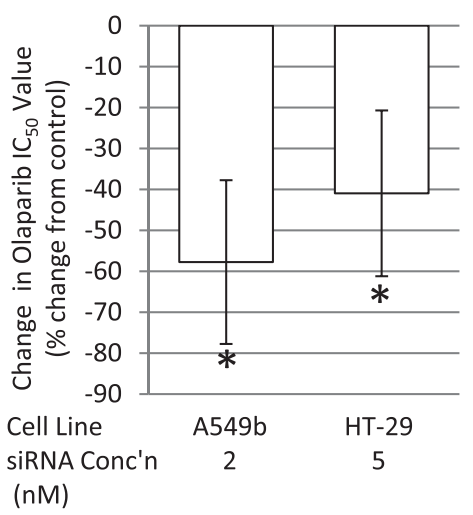

A

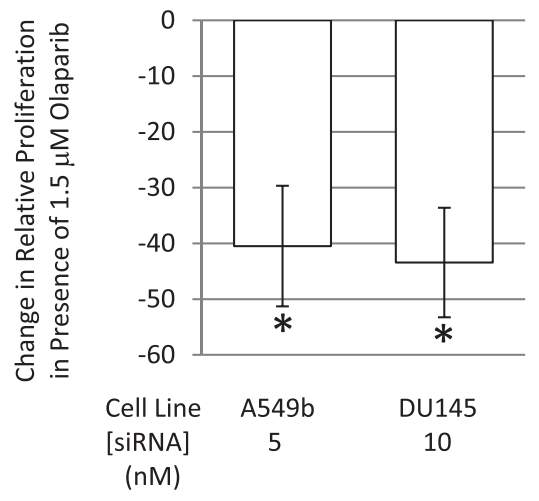

B

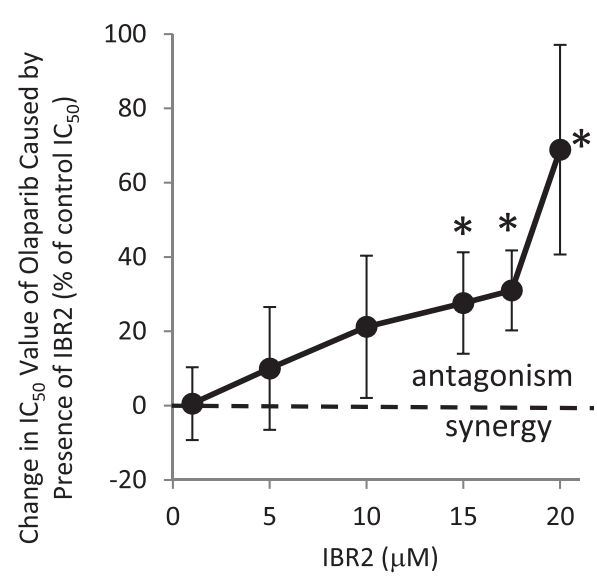

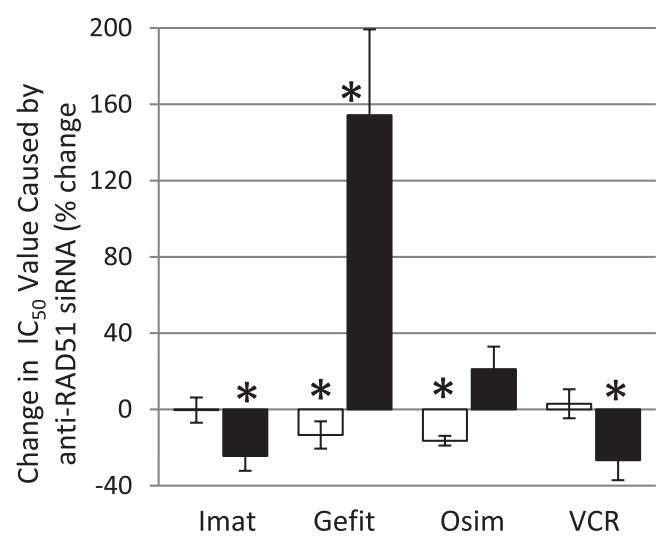

C

Fig. 7. Comparison of the effect of RAD51 antisense molecule and RAD51 inhibitor IBR2 on the cytotoxicity of olaparib and other anticancer drugs. (A, C, and D) Cells were cultured in $25-\mathrm{cm}^{2}$ flasks, and exposed to siRNA as described in Materials and Methods for 4 hours, followed by addition of one volume of medium. After a further 20 hours, the medium was changed and the drug indicated was added to the final desired concentration. The proliferation of cells in the presence of the indicated concentration of siRNA, as a percentage of the scrambled siRNA control, in (A) was $85.3 \% \pm 11.0 \%(n=9)$ for A549b and $70.2 \% \pm 11.9 \%$ (7) for HT-29. In $(\mathrm{C}$ and $\mathrm{D})$ the proliferation was $51.8 \% \pm$ $12.7 \%$ (8) for A549b and $62.4 \% \pm 11.6 \%$ (9) for DU145. (B) A549b cells were grown in 96-well plates and treated with simultaneous exposure to IBR2 and olaparib at the concentrations indicated. The relative cell density was determined after 4 days by viability staining. The relative density of cells treated with both agents was normalized to the density observed for the respectively treated IBR2 control. The $\mathrm{IC}_{50}$ value was interpolated from plotted data. The change in the $\mathrm{IC}_{50}$ value caused by anti-RAD51 antisense or the presence of IBR2 was determined as a percentage of the $\mathrm{IC}_{50}$ value of drug in the absence of cotreatment. Negative values indicate synergy, whereas positive values indicate antagonism. $* P<0.05$. Gefit, gefitinib; Imat, imatinib; Osim, osimertinib; VCR, vincristine.
$\mathrm{HN}-5 \mathrm{a} / \mathrm{V} 15 \mathrm{e}$ was not cross resistant to imatinib or IBR2 (Fig. 1), suggesting that Pgp does not modulate imatinib activity in this cell line. [In contrast, imatinib has been reported to be a Pgp substrate in other cells (Radujkovic et al., 2005)]. IBR2 enhanced imatinib toxicity in $\mathrm{HN}-5 \mathrm{a} / \mathrm{V} 15 \mathrm{e}$, its parent line HN-5a, and HT-29 by up to $60 \%$, much better than verapamil (up to $40 \%$ at similar concentrations, $P<$ 0.05). IBR2 enhanced vincristine toxicity in these three cell lines to a degree similar to verapamil, decreasing the $\mathrm{IC}_{50}$ value by up to $90 \%$. Therefore, there is no conclusive evidence that IBR2 interacts with Pgp to enhance drug cytotoxicity.

5. Another RAD51 inhibitor, B02, displayed similar enhancement of imatinib and vincristine as IBR2 (Huang et al., 2012). Although this molecule may likewise not necessarily be specific for RAD51, it suggests an overlapping mechanism of enhancement of drug toxicity by two compounds that were designed as specific inhibitors of RAD51.

6. The PPAR $\gamma$ inhibitor T007 decreases $\alpha$ and $\beta$ tubulin proteins in some cancer cell lines and also appears to inhibit RAD51 (An et al., 2016). This suggests that inhibitors of DNA repair, possibly including a RAD51 inhibitor, may influence the activity of microtubules, and may contribute to the sensitization of cell lines to vincristine, as reported herein.

7. Modulators of protein turnover decrease levels of RAD51 as well as various proteins involved in signal transduction and DNA repair (Ko et al., 2012; Smyth et al., 2012; Segawa et al., 2014).

8. Bcr-Abl upregulates RAD51 (Slupianek et al., 2001; Noguchi et al., 2006; Suhane et al., 2015; Jiang et al., 2017). Therefore, in a cell line such as K562 which is dependent on Bcr-Abl as its oncogenic driver, the BcrAbl inhibitor imatinib could potentially cause a decrease in RAD51, increasing sensitivity to IBR2.

9. IBR2 generally enhanced activity of inhibitors of EGFR. The exception was erlotinib, for which the interaction ranged from synergistic to nil to antagonistic.

10. Akt and EGFR are both suggested to regulate the level of RAD51 (Ko et al., 2016; Zhong et al., 2016). Therefore, an inhibitor of EGFR could potentially decrease the level of RAD51, making the cell more sensitive to a RAD51 inhibitor.

11. For the following reasons, Hsp90 was considered as a possible secondary target for IBR2: 1) inhibition of Hsp90 (A549 and H1975) or its yeast ortholog heat shock protein 82 results in decreased RAD51 (degradation by proteosomes) and inhibits DNA repair (Ko et al., 2012; Segawa et al., 2014; Suhane et al., 2015); 2) the Hsp90 inhibitor $17-N$-allylamino17-demethoxygeldanamycin (17-AAG) downregulated Bcr-Abl protein level in CML cells, and acted synergistically with imatinib to kill Bcr-Abl-overexpressing CML cells (Radujkovic et al., 2005); 3) Hsp90 is a chaperone for EGFR and Bcr-Abl (Jhaveri et al., 2014); and 4) inhibition of Hsp90 sensitizes human lung 
cancer cell lines to treatment with inhibitors of EGFR (Courtin et al., 2016). Therefore, the Hsp90 inhibitor 17-AAG was tested in combination with several drugs against various cell lines (Supplemental Figs. 10-13). 17-AAG, which is cytotoxic in the nanomolar range (Supplemental Fig. 10), was not synergistic with imatinib, osimertinib, or vincristine, but showed good synergy with gefitinib at most concentrations, except against HEK293 (Supplemental Figs. 12-13). The minimal synergy between $17-\mathrm{AAG}$ and a range of drugs tends to preclude Hsp90 as a secondary target of IBR2.

12. The inability of antisense against RAD51 to recapitulate the synergy between IBR2 and four chemotherapy drugs suggests that IBR2 may have secondary targets and/or requires the presence of RAD51 to create an inhibitory entity.

Notwithstanding these observations, it remains possible that interaction of IBR2 with RAD51 or a nonspecific target has a common node of interactions among the various targets of the drugs assayed herein. IBR2 was most effective in sensitizing human cancer cell lines to treatment with imatinib, regorafenib, EGFR inhibitors (including erlotinib, gefitinib, afatinib, and osimertinib), and vincristine. It may have less potential for useful combination with olaparib, melphalan, cisplatin, paclitaxel (in non-Pgp-overexpressing tumors), or irinotecan. The potential for IBR2 to induce synthetic lethality and increase selectivity of anticancer treatment, sparing normal tissues and improving the overall success of numerous treatments, is worthwhile to investigate in animal models.

\section{Acknowledgments}

We thank Ronak Zareardalan for technical assistance with the cytotoxicity assays and Dr. Saman Maleki and Dr. Kristin Chadwick for technical assistance with the flow cytometry. This work is dedicated to the memory of Mary Ferguson.

\section{Authorship Contributions}

Participated in research design: Ferguson, Koropatnick, Vincent. Conducted experiments: Ferguson.

Performed data analysis: Ferguson.

Wrote or contributed to the writing of the manuscript: Ferguson, Koropatnick, Vincent.

\section{References}

An Z, Yu JR, and Park WY (2016) T0070907 inhibits repair of radiation-induced DNA damage by targeting RAD51. Toxicol In Vitro 37:1-8.

Balbous A, Cortes U, Guilloteau K, Rivet P, Pinel B, Duchesne M, Godet J, Boissonnade O, Wager M, Bensadoun RJ, et al. (2016) A radiosensitizing effect of RAD51 inhibition in glioblastoma stem-like cells. BMC Cancer 16:604.

Beghini A, Bellini M, Magnani I, Colapietro P, Cairoli R, Morra E, and Larizza L (2005) STI 571 inhibition effect on KIT ${ }^{\text {Asn822Lys }}$-mediated signal transduction cascade. Exp Hematol 33:682-688.

Blake A, Dragan M, Tirona RG, Hardy DB, Brackstone M, Tuck AB, Babwah AV, and Bhattacharya M (2017) G protein-coupled KISS1 receptor is overexpressed in triple negative breast cancer and promotes drug resistance. Sci Rep 7:46525.

Bryant HE, Schultz N, Thomas HD, Parker KM, Flower D, Lopez E, Kyle S, Meuth M, Curtin NJ, and Helleday T (2005) Specific killing of BRCA2-deficient tumours with inhibitors of poly(ADP-ribose) polymerase. Nature 434:913-917.

Courtin A, Smyth T, Hearn K, Saini HK, Thompson NT, Lyons JF, and Wallis NG (2016) Emergence of resistance to tyrosine kinase inhibitors in non-small-cell lung cancer can be delayed by an upfront combination with the HSP90 inhibitor onalespib. Br J Cancer 115:1069-1077.

Farmer H, McCabe N, Lord CJ, Tutt ANJ, Johnson DA, Richardson TB, Santarosa M Dillon KJ, Hickson I, Knights C, et al. (2005) Targeting the DNA repair defect in BRCA mutant cells as a therapeutic strategy. Nature 434:917-921.

Ferguson PJ, Brisson AR, Koropatnick J, and Vincent MD (2009) Enhancement of cytotoxicity of natural product drugs against multidrug resistant variant cell lines of human head and neck squamous cell carcinoma and breast carcinoma by tesmilifene. Cancer Lett 274:279-289.

Ferguson PJ, Rytelewski M, Vincent MD, Figueredo R, and Koropatnick J (2013) Sensitization of human tumor cells to chemotherapy drugs by antisense downregulation of BRCA2 and thymidylate synthase (TS): induction of synthetic lethality by targeting DNA repair (Abstract). Proc Amer Assoc Cancer Res 54:3314.

Ferguson PJ, Rytelewski M, Vincent MD, and Koropatnick J (2016) Sensitization of human tumor cells to chemotherapy drugs by antisense downregulation of RAD51: targeting DNA repair to induce synthetic lethality (Abstract). Proc Amer Assoc Cancer Res 57:3718.

Godin SK, Sullivan MR, and Bernstein KA (2016) Novel insights into RAD51 activity and regulation during homologous recombination and DNA replication. Biochem Cell Biol 94:407-418.

Huang F, Mazina OM, Zentner IJ, Cocklin S, and Mazin AV (2012) Inhibition of homologous recombination in human cells by targeting RAD51 recombinase. $J \mathrm{Med}$ Chem 55:3011-3020.

Jhaveri K, Ochiana SO, Dunphy MP, Gerecitano JF, Corben AD, Peter RI, Janjigian YY, Gomes-DaGama EM, Koren J, III, Modi S, et al. (2014) Heat shock protein 90 inhibitors in the treatment of cancer: current status and future directions. Expert Opin Investig Drugs 23:611-628.

Jiang J, Lu Y, Li Z, Li L, Niu D, Xu W, Liu J, Fu L, Zhou Z, Gu Y, et al. (2017) Ganetespib overcomes resistance to PARP inhibitors in breast cancer by targeting core proteins in the DNA repair machinery. Invest New Drugs 35:251-259.

Ko JC, Chen HJ, Huang YC, Tseng SC, Weng SH, Wo TY, Huang YJ, Chiu HC, Tsai MS, Chiou RY, et al. (2012) HSP90 inhibition induces cytotoxicity via downregulation of Rad51 expression and DNA repair capacity in non-small cell lung cancer cells. Regul Toxicol Pharmacol 64:415-424.

Ko JC, Chen JC, Wang TJ, Zheng HY, Chen WC, Chang PY, and Lin YW (2016 Astaxanthin down-regulates Rad51 expression via inactivation of AKT kinase to enhance mitomycin C-induced cytotoxicity in human non-small cell lung cancer cells. Biochem Pharmacol 105:91-100.

Lapointe H, Lampe H, and Banerjee D (1992) Head and neck squamous cell carcinoma cell line-induced suppression of in vitro lymphocyte proliferative responses. Otolaryngol Head Neck Surg 106:149-158.

Lee WH, Chen PL, and Zhu J (2009) inventors. Compositions and methods for disruption of BRCA2-RAD51 interaction. U.S. patent 2009/0221634 A1. Assignee: The Regents of the University of California, Oakland, CA. Publication date: September 3, 2009

Lv W, Budke B, Pawlowski M, Connell PP, and Kozikowski AP (2016) Development of small molecules that specifically inhibit the D-loop activity of RAD51. J Med Chem 59:4511-4525.

Noguchi M, Yu D, Hirayama R, Ninomiya Y, Sekine E, Kubota N, Ando K, and Okayasu $R$ (2006) Inhibition of homologous recombination repair in irradiated tumor cells pretreated with Hsp90 inhibitor 17-allylamino-17-demethoxygeldanamycin. Biochem Biophys Res Commun 351:658-663.

Radujkovic A, Schad M, Topaly J, Veldwijk MR, Laufs S, Schultheis BS, Jauch A, Melo JV, Fruehauf S, and Zeller WJ (2005) Synergistic activity of imatinib and 17-AAG in imatinib-resistant CML cells overexpressing BCR-ABL-inhibition of P-glycoprotein function by 17-AAG. Leukemia 19:1198-1206.

Rytelewski M, Ferguson PJ, Maleki Vareki S, Figueredo R, Vincent M, and Koropatnick J (2013) Inhibition of BRCA2 and thymidylate synthase creates multidrug sensitive tumor cells via the induction of combined "complementary lethality". Molec Ther Nucl Acids 2:e78.

Rytelewski M, Maleki Vareki S, Mangala LS, Romanow L, Jiang D, Pradeep S, Rodriguez-Aguayo C, Lopez-Berestein G, Figueredo R, Ferguson PJ, et al. (2016) Reciprocal positive selection for weakness-preventing olaparib resistance by inhibiting BRCA2. Oncotarget 7:20825-20839.

Segawa T, Fujii Y, Tanaka A, Bando S, Okayasu R, Ohnishi K, and Kubota N (2014) Radiosensitization of human lung cancer cells by the novel purine-scaffold Hsp90 inhibitor, PU-H71. Int J Mol Med 33:559-564.

Slovak ML, Ho JP, Bhardwaj G, Kurz EU, Deeley RG, and Cole SP (1993) Localization of a novel multidrug resistance-associated gene in the HT1080/DR4 and H69AR human tumor cell lines. Cancer Res 53:3221-3225.

Slupianek A, Schmutte C, Tombline G, Nieborowska-Skorska M, Hoser G, Nowicki MO, Pierce AJ, Fishel R, and Skorski T (2001) BCR/ABL regulates mammalian RecA homologs, resulting in drug resistance. Mol Cell 8:795-806.

Smyth T, Van Looy T, Curry JE, Rodriguez-Lopez AM, Wozniak A, Zhu M, Donsky R Morgan JG, Mayeda M, Fletcher JA, et al. (2012) The HSP90 inhibitor, AT13387, is effective against imatinib-sensitive and -resistant gastrointestinal stromal tumor models. Mol Cancer Ther 11:1799-1808.

Suhane T, Laskar S, Advani S, Roy N, Varunan S, Bhattacharyya D, Bhattacharyya S, and Bhattacharyya MK (2015) Both the charged linker region and ATPase domain of Hsp90 are essential for Rad51-dependent DNA repair. Eukaryot Cell 14: 64-77.

Wiegmans AP, Miranda M, Wen SW, Al-Ejeh F, and Möller A (2016) RAD51 inhibition in triple negative breast cancer cells is challenged by compensatory survival signaling and requires rational combination therapy. Oncotarget 7: 60087-60100.

Zhong X, Luo G, Zhou X, Luo W, Wu X, Zhong R, Wang Y, Xu F, and Wang J (2016) Rad51 in regulating the radiosensitivity of non-small cell lung cancer with different epidermal growth factor receptor mutation status. Thorac Cancer 7:50-60.

Zhu J, Zhou L, Wu G, Konig H, Lin X, Li G, Qiu XL, Chen CF, Hu CM, Goldblatt E, et al. (2013) A novel small molecule RAD51 inactivator overcomes imatinibresistance in chronic myeloid leukaemia. EMBO Mol Med 5:353-365.

Address correspondence to: Peter Ferguson, Cancer Research Laboratory Program - London Regional Cancer Program, London Health Sciences Centre, 790 Commissioners Road East, London, Ontario N6A 4L6, Canada. E-mail: peter.ferguson@uwo.ca 